\title{
Linx
}

Revue des linguistes de l'université Paris X Nanterre

$55 \mid 2006$

Thème et thématisation

\section{Thématisation dans les langues flexionnelles : le cas du russe}

Serguei Sakhno

\section{OpenEdition}

Journals

Édition électronique

URL : http://journals.openedition.org/linx/372

DOI : $10.4000 / \operatorname{linx} .372$

ISSN : 2118-9692

Éditeur

Presses universitaires de Paris Nanterre

\section{Édition imprimée}

Date de publication : 1 décembre 2006

Pagination : 15-31

ISSN : 0246-8743

\section{Référence électronique}

Serguei Sakhno, «Thématisation dans les langues flexionnelles : le cas du russe », Linx [En ligne], 55 | 2006, mis en ligne le 25 février 2011, consulté le 01 mai 2019. URL : http:// journals.openedition.org/linx/372 ; DOI : 10.4000/linx.372 


\title{
Thématisation dans les langues flexionnelles : le cas du russe
}

\author{
Sergueï Sakbno \\ Département de russe et MoDyCo, \\ U.Paris 10, ssakbno@u-paris10.fr
}

Pour une langue comme le russe, parler de marques de thématisation n'a de sens que si l'on tient compte de toute la corrélation «thème / rhème », cette corrélation étant - ne l'oublions pas - de nature informationnelle, communicative (Janko, 2001, p. 23-25), et des différentes façons de définir ce phénomène assez complexe qui a fait l'objet d'une littérature immense, notamment en slavistique, dont il est impossible de faire ici le résumé ni même un aperçu succinct. Il existe des travaux assez précis et détaillés sur cet aspect du russe désigné également par le terme (souvent utilisé) aktual'noe členenie predloženija «division actuelle de la phrase» (Benoist, 1979, Kovtunova, 1976, 1980, Zolotova, 1982, Fougeron, 1989, Bonnot, 1999). Notons en particulier l'ouvrage récent de T. Janko (2001), qui synthétise bien l'état de la question tout en apportant des analyses éclairantes et des éléments nouveaux sur la relation thème / rhème en tant qu'inscrite dans les "stratégies communicatives » du russe ${ }^{1}$.

Le cadre restreint de cet article ne permet guère de décrire de façon tant soit peu satisfaisante les principaux phénomènes liés à la thématisation en russe. Nous

\footnotetext{
${ }^{1}$ Je tiens à remercier Mme Tatiana Janko (Institut de linguistique, Moscou) pour sa relecture attentive et compétente de la version initiale de mon texte ainsi que pour ses remarques qui ont contribué à l'améliorer.
} 
nous limitons à quelques-uns parmi les faits majeurs en les abordant essentiellement d'un point de vue didactique, dans la mesure où ils constituent une spécificité du russe par rapport au français. Ces faits relèvent de trois niveaux (Gak, 1989, p. 208) :

- $\quad$ niveau syntaxique : changements dans l'ordre des mots ;

- niveau prosodique: intonation (ton, intensité, débit, accent de phrase, possibilité de pause entre le thème et le rhème);

- $\quad$ niveau lexical : particules et autres mots du discours.

Cette partition est certes conventionnelle : d'une part, il est difficile de séparer le prosodique du syntaxique (ainsi, le phénomène de l'intonation, dont le rôle dans le découpage thème-rhème est essentiel, relève de la prosodie, mais il est en rapport avec l'ordre des mots), d'autre part, le fonctionnement des mots marqueurs du thème ou du rhème est étroitement lié à certaines structures syntaxiques aussi bien qu'à des faits de prosodie.

Pour faciliter la présentation des faits du russe, nous nous sommes contraint à illustrer notre propos par des séquences simples, en excluant par exemple les structures comportant un Nominatif, un Accusatif et un Datif, du type Ivan (Nom.) daët knigu (Acc.) synu (Dat.) 'Ivan donne un livre à (son) fils'.

\section{Division actuelle de la phrase, mobilité de l'ordre des mots et flexion}

La distinction «thème / rhème » fut élaborée à la fin des années 1920 par l'École de Prague, notamment par V. Mathesius. J.-P. Benoist (1979) rappelle que l'idée remonte à Henri Weil qui observa dans sa thèse de doctorat (1844) que l'ordre des mots était dans les langues classiques (langues à flexion) le moyen d'expression de la marche de la pensée qui procède d'une idée connue (" point de départ », «notion initiale ») pour aboutir à une idée essentielle (« but du discours », " énonciation »).

Si ce principe se réalise avec le plus d'évidence dans les langues à flexion, c'est parce que la flexion casuelle présuppose une relative liberté de l'ordre des mots : plus exactement, l'ordre des mots, étant dégagé des obligations syntaxiques formelles liées au marquage des positions syntaxiques essentielles (sujet, verbe, complément d'objet, etc.), offre la possibilité de jouer sur l'ordre des mots à des fins communicatives et expressives.

C'est le cas du russe et d'autres langues à flexion nominale considérable, notamment, des langues classiques (grec, latin), des langues slaves comme le tchèque, langue maternelle de plusieurs linguistes de l'Ecole de Prague, ou le polonais (cf. Adamczewski, 1991, p. 211), ou de celles à flexion nominale résiduelle (ancien français, cf. Marchello-Nizia, 1999, p. 38-48).

Ainsi, dans les énoncés suivants, la flexion nominale (notamment, la désinence $-a$ qui marque l'Accusatif de syn 'fils') permet d'identifier le sujet et le COD et de maintenir la même structure actancielle tout en changeant l'ordre des constituants et en modifiant la structure thémo-rhématique : 
Th $\quad \mathrm{Rh}$

'Ivan réveille (son) fils', mot à mot «Ivan (Nominatif) réveille (Prés., 3e pers. sing.) fils (Accusatif sing. masculin animé)»

Syna (Accusatif) budit // Ivan (Nominatif)

Th

$\mathrm{Rh}$

'Le fils, c'est Ivan qui le réveille / Le fils est réveillé par Ivan', mot à mot «Fils (Accusatif sing. masculin animé) réveille (Prés., $3^{e}$ pers. sing.) Ivan (Nominatif)»

Les traductions françaises possibles du dernier exemple montrent que le français obtient un effet de thématisation proche par d'autres procédés (dislocation syntaxique avec reprise anaphorique ; passivation).

Il faut préciser cependant que l'on distingue dans la phrase russe termes primaires et termes secondaires : les termes primaires correspondent au sujet, au verbe, aux compléments du verbe, à l'attribut du sujet ou du complément d'objet direct et aux déterminants du verbe ; les termes secondaires correspondent aux autres éléments de la phrase. Seuls les termes primaires peuvent être disposés librement, alors que les termes secondaires sont dans un ordre fixe par rapport au terme primaire dont ils dépendent, ainsi le complément du nom qui est normalement postposé : dans le discours non poétique, on aurait dom Ivana 'la maison d'Ivan' plutôt que ??Ivana dom (Comtet, 2002, p. 400).

\section{Flexion nominale en russe : marques, oppositions et cas d'homonymie grammaticale}

Il convient de souligner que la nature flexionnelle du russe est assez marquée dans le domaine du nom: ayant préservé (certes, avec des modifications et des simplifications) l'essentiel du système casuel indo-européen, le russe dispose de 6 cas (Nominatif, Accusatif, Génitif, Datif, Prépositif, Instrumental), les substantifs se répartissent en 3 déclinaisons (dont chacune avec des sous-types). L'adjectif, le pronom et le déterminant se déclinent également.

Le russe a même développé de nouvelles formes et des oppositions casuelles supplémentaires. Parmi les faits les plus spectaculaires, notons :

a) apparition progressive d'un Accusatif «animé » pour les masculins dès le vieux russe ${ }^{2}$;

2 Autrefois, il y a dix siècles environ, l'Accusatif de ces substantifs était identique au Nominatif. Quand on disait Ivanŭ (Nom. = Acc.) budiť synŭ (Nom. = Acc.) 'Ivan réveille (son) fils' / Le fils réveille Ivan', la phrase était gênante à cause de son ambiguité : on ne savait pas trop où était le sujet et où était le COD. Pour pallier cette confusion, la langue a cherché une solution : l'Accusatif des noms masculins animés devait être différent du Nominatif. Pour marquer le COD, on a fini par adopter une forme coïncidant avec le Génitif : Ivanŭ (Nom.) budiť̌ synŭ / syna (Acc. "animé »= 
b) formation en russe moderne, pour certains substantifs masculins, de deux Génitifs et deux Locatifs à valeurs distinctes, grâce à la récupération de variantes désinentielles dues aux vestiges d'anciennes déclinaisons en ${ }^{\circ}$ os et en *ŭs. Ainsi le Prépositif qui se divise en 2 cas : Prépositif tout court (ou Prép.1) videt' simvoližm v lese 'voir un symbolisme dans la forêt (comme concept)' et Locatif (ou Prép.2) guljat'v lesu 'se promener dans la forêt (comme espace)'.

Toutefois, pour certains modèles de déclinaisons, la flexion n'est pas suffisamment discriminante, lorsque par exemple la forme du Nominatif coïncide avec celle de l'Accusatif, ce qui en l'absence de contexte entraîne une certaine ambiguïté syntaxique pouvant aboutir à la nécessité d'un ordre des mots plus contraint (Benoist 1979, p. 30, Comtet, 2002, p. 402). Les grammairiens considèrent souvent que le russe tendrait dans ce cas à limiter la mobilité de l'ordre des mots en adoptant l'ordre direct à la française, de type SVO :

(3) Mat' budit doč'

'La mère réveille (sa) fille',

mot à mot « Mère (Nom. = Acc.) réveille (Prés., $3^{e}$ pers. sing.) fille (Nom. = Acc.)»

Mais si cette phrase est contextualisée comme réponse à la question :

(4) Kto budit mat'?

'Qui / qui est-ce qui réveille la mère?',

il devient possible qu'en discours réel, mat' soit interprété comme un COD en position de thème frontal :

(3') Mat' budit // doč'

Th $\mathrm{Rh}$

'C’est la fille qui réveille (sa) mère / La mère est réveillée par sa fille'.

Par ailleurs, l'Accusatif du COD peut être marqué en cas de besoin au niveau d'un déterminant, par exemple grâce à la désinence de l'adjectif possessif réfléchi qui oppose svoja (Nom. sing. fém.) à svoju (Acc. sing. fém.) :

Svoju mat' budit // doč'

Th $\mathrm{Rh}$

'C'est la fille qui réveille sa mère', mot à mot « Sa (Acc. sing. fém.) mère réveille / / fille »

Gén.) 'Ivan réveille (son) fils'. En revanche, les inanimés n’ont pas subi ce type de changement : Ivanŭ (Nom. = Acc.) vidiť stolŭ $($ Nom. $=$ Acc.) 'Ivan voit une table' n'était pas ambigu. Le problème ne s'est jamais posé pour les féminins en $-a$ grâce à la parfaite distinction entre le Nominatif et l'Accusatif, cf. Anna (Nom.) budiť̌ Irinu (Acc.) 'Anna réveille Irina'. 
De toute façon, ce phénomène d'homonymie morphologique reste relativement marginal (les féminins de la $3^{e}$ déclinaison n'étant pas très nombreux), comme le sont d'ailleurs les rares phrases potentiellement ambiguës hors contexte, comportant des masculins inanimés et / ou des neutres ${ }^{3}$.

Un exemple particulier comportant deux substantifs neutres mérite d'être cité : il s'agit d'un des adages de la «philosophie marxiste-léniniste » que l'on apprenait dès le lycée à l'époque soviétique :

\section{(5) Bytie opredeljaet soznanie}

'L'existence matérielle détermine la conscience'.

Il était normal de considérer bytie comme sujet syntaxique pour des raisons linguistiques: difficulté de contextualiser ce genre de maxime dans un contexte de dialogue réel, d'où la quasi impossibilité d'imaginer une situation de communication où bytie serait traité comme COD placé en position de thème frontal ; ordre SVO prescrit par la grammaire scolaire en cas d'ambiguité structurelle. Mais c'est surtout en raison de l'aspect idéologique de l'aphorisme (principe du matérialisme dialectique) qu'il était impensable de traiter cette phrase comme ambiguë. Dans le cas contraire, on risquait d'être taxé d'idéaliste, voire d'« élément hostile manquant de maturité idéologique et politique »!

\section{Division actuelle de la phrase et détermination du GN}

Un autre phénomène important découle de la corrélation «thème / rhème » rendue par l'ordre des mots : dans la mesure où le russe, tout comme les autres langues slaves (à l'exception notable du bulgare et du macédonien) ne connait pas l'article, l'ordre des mots y permet souvent de rendre ce qui correspond, dans les langues à article, à la détermination ou l'indétermination du substantif :

(6) Student - tut

Th $\mathrm{Rh}$

'L'étudiant (bien déterminé, connu) est ici'

(7) Tut-student

Th $\mathrm{Rh}$

'Ici est / se trouve un étudiant (indéterminé, inconnu, un parmi d'autres)'

Cette régularité est à prendre en compte lors la traduction du français vers le russe (exemple cité dans Gak, 1989, p. 211) :

(8) Après un peu de temps une petite sonnerie a résonné dans la pièce (A. Camus, L'étranger)

Nemnogo spustja v zale zijaknul zvonok

(trad. N. Gal), mot à mot «Un peu après dans pièce résonna sonnerie ».

${ }^{3}$ Cf. Gruzovik obognal avtomobil' qui s'interprète hors contexte comme 'Le camion a doublé la voiture' (cf. exemple proche dans Comtet, 2002, p. 402). 
Elle est souvent observée lors de la traduction du russe vers le français (exemples pris dans Boulanger, 2000, p. 146) :

(9) V priëmnoj menja そ̌dala molodaja ženščina

Une jeune femme m'attendait à l'accueil.

(10) Uvidev menja, molodaja ženščina vstala

Quand elle me vit, la jeune femme se leva.

\section{Complexité discursive de la notion de thème}

Certains spécialistes notent la difficulté d'identifier le thème dans plusieurs contextes. On a souvent tendance à chercher d'abord le rhème, réputé plus « marqué ", le reste devant être considéré comme thème (Nikolaeva, 2000, p. 70) ; certains travaux d'analyse textuelle s'intéressent en effet au rhème au détriment du thème (Zolotova, 1982, p. 300-319).

On remarque notamment que la notion de thème ne correspond pas toujours exactement à celle de «connu » ni à celle de «donné » ${ }^{4}$. Selon I. Kovtunova (1980 : 193), dans certains cas, le thème peut comporter des éléments d'information nouvelle. Pour illustrer ce phénomène, elle cite un exemple tiré d'un texte où l'élément skazki 'contes' fait partie du thème, quoiqu'il soit mentionné pour la première fois :

(11) Skąki njanja nam // ne rasskazyvala (V. Šklovskij)

Th $\quad \mathrm{Rh}$

'La nounou ne nous disait pas de contes', mot à mot «Contes nounou nous

(Datif) // ne racontait (Passé imperf.) ».

Nous adhérons à l'idée de la complexité potentielle du thème pouvant comporter du «nouveau ». Mais cette analyse de (11) nous paraît discutable : tout dépend de la façon dont on définit la «nouveauté » de l'information. Au niveau textuel, l'élément skąki ne relève pas vraiment du « nouveau » à cause des présuppositions convoquées par njanja 'nounou' dans le cadre d'un récit où le narrateur se souvient de son enfance. En Russie, l'image traditionnelle de la nounou de l'époque décrite par le texte en question est celle d'une femme qui connait des contes populaires et qui aime les dire souvent aux enfants dont elle s'occupe.

Or il est vrai que dans certains contextes dialogiques, on peut avoir une situation paradoxale, où le thème correspond à une information véritablement nouvelle pour l'interlocuteur, alors que le rhème correspond au contraire à une information donnée (exemple de Janko, 2001, p. 30) :

\footnotetext{
${ }^{4}$ A la suite de W. Chafe (1976), il convient de distinguer ces deux notions : le « donné » est l'élément que le locuteur suppose présent dans l'esprit de son interlocuteur au moment de l'énonciation, le « connu » est l'élément faisant partie de l'ensemble des connaissances que le locuteur suppose chez son interlocuteur.
} 


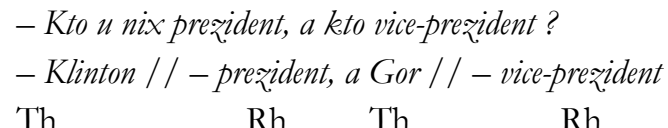

'- Qui est chez eux / là-bas président, et qui est vice-président ?

- Clinton est président, alors que Gore est vice-président'.

Certes, cet exemple a un caractère particulier, puisqu'il s'agit d'un choix contraint lié à la superposition de deux schémas énonciatifs (il faut dire qui est qui dans le tandem du sommet exécutif américain tout en l'identifiant) et que le locuteur fait comme si les noms de Clinton et de Gore devaient être connus de l'interlocuteur ${ }^{5}$. Mais il montre que le thème ne peut pas être toujours défini à partir de critères pertinents ("donné » $v S$ " nouveau ») mais pris de façon trop absolue.

Le thème est en fait une construction discursive (voir des points de vue proches dans Guimier, 1999) qui s'inscrit dans telle ou telle «stratégie communicative » (selon Janko, 2001, c'est d'ailleurs le mot clé de son ouvrage).

Dans un schéma communicatif davantage classique - et qui s'avère en même temps bien plus « plat» du point de vue discursif - on aurait :

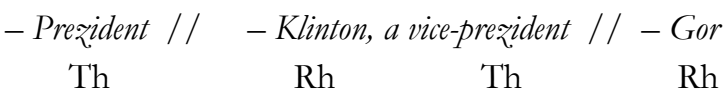

\section{Thème frontal en syntaxe russe dite neutre : aspects linguistiques et pédagogiques}

Ce qui frappe un francophone qui aborde le russe, c'est la liberté structurelle qu'offre cette langue pour transformer en thème tout élément par le simple fait de le placer en position frontale dans une phrase. Dans des situations normales de communication, c'est-à-dire en dehors des schémas émotifs et rhétoriques, le thème précède le rhème dans la langue standard : le locuteur passe du "connu» au "nouveau», du support de l'information à l'information qui est communiquée à propos de ce support. Il s'agit d'un ordre non marqué dit aussi «neutre», «normal», "grammatical », par opposition à l'ordre inversé (rhème - thème) qui est considéré comme l'ordre marqué, «expressif », « emphatique ».

Ce principe simple est d'une importance pédagogique capitale pour saisir le fonctionnement du russe et pour faire comprendre à un étudiant pourquoi il est préférable de répondre à une question comme

Kto ljubit Annu?

'Qui / qui est-ce qui aime Anna?'

par:

\footnotetext{
${ }^{5}$ Ce qui est proche du procédé rhétorique qu'utilisent les enseignants : En 1920, la poétesse russe Akhmatova - que vous connaissez certainement.../ dont le nom est connu de tous... (même si le locuteur a toutes les raisons de penser que les étudiants ignorent le nom de cette poétesse).
} 
Annu ljubit // Viktor

Th

$\mathrm{Rh}$

(la limite thémo-rhématique correspondant à une pause éventuelle est notée par //)

'Anna, c'est Victor qui l'aime', mot à mot « Anna (Accusatif) aime Victor (Nominatif)» plutôt que par

(16) Viktor ljubit Annu

'Victor aime Anna'

(exemple tiré de notre enseignement du russe grands débutants à l'Université Paris 10).

En classe de langue, l'explication ne passe pas toujours facilement. Répondant à la question (14), la plupart des étudiants francophones préfèrent à (15) la variante (16) Viktor ljubit Annu qui est plus conforme au schéma syntaxique normal du français écrit - mais qui serait incorrecte en russe du point de vue informationnel car elle suppose une interrogation du genre

\section{(17) Kogo ljubit Viktor ? 'Qui Victor aime-t-il ?'.}

L'analogie avec une construction française disloquée avec reprise anaphorique, utilisée parfois comme repère pédagogique afin de faire saisir la logique de la phrase russe (15) aux étudiants, n'est pas très opérante, puisque les phrases françaises relèvent d'un schéma syntaxique tout différent: Anna, celui qui l'aime, c'est Victor; Anna, c'est Victor qui l'aime.

Inversement, un étudiant russophone aura tendance à transposer de façon mécanique cette particularité du russe sur le français, en produisant des énoncés tels que

*Le professeur // nous avons attendu longtemps ${ }^{6}$

Th

$\mathrm{Rh}$

(cf. russe Prepodavatelja // my ždali dolgo)

au lieu du schéma français normal avec dislocation et reprise anaphorique

(19) Le professeur, nous l'avons attendu longtemps.

\subsection{Prédicat comme thème frontal}

Même un prédicat peut être thématisé par sa mise en position frontale, avec une aisance syntaxique inhabituelle pour un francophone, comme c'est le cas dans la séquence dialogique suivante :

\footnotetext{
${ }^{6}$ Ce phénomène dû à l'interférence linguistique n'est pas à confondre avec certains faits de rhématisation chez des francophones natifs. En effet, la syntaxe du français oral non standard autorise la position frontale du rhème avec accent emphatique (focus intonatif) en fonction de rhème : Mardi il faut attendre! (énoncé entendu dans la bouche d'un francophone) ou Les noires je prends (une cliente dans un magasin de chaussures, exemple cité dans Sitri, 2003, p. 45).
} 
(20) - Kto igraet na gitare? A keto poët? Viktor ili Anna?

- Igraet // Viktor, a poët // Anna.

Th $\quad \mathrm{Rh}$ Th $\mathrm{Rh}$

- 'Qui joue de la guitare? Et qui chante ? Est-ce Victor ou Anna ?'

- 'C'est Victor qui joue, et c'est Anna qui chante / Celui qui joue c'est Victor, et celle qui chante c'est Anna', mot à mot «Joue Victor, et chante Anna »

S'il est mal compris, ce phénomène rend parfois difficile la compréhension d'un texte écrit, par exemple d'un article de presse. Voici un cas anecdotique (tiré de notre expérience pédagogique). Un article consacré aux péripéties de la politique ukrainienne commençait par :

Opjat' pobedil / / Juššenko

Th

$\mathrm{Rh}$

'C'est Youchtchenko qui a encore vaincu / qui a encore gagné' / La victoire revient de nouveau à Youchtchenko', mot à mot « De nouveau vainquit (Passé perfectif) Youchtchenko »

Un étudiant a été dérouté par la position du verbe situé à gauche du sujet et précédé de l'adverbe opjat' 'de nouveau, encore' (en russe, l'adverbe se place souvent avant le verbe). De plus, il ignorait le sens de opjat', et il a été tenté de traiter ce dernier comme un nom propre inconnu en position de sujet. Il a alors traduit: Opiat a vaincu Youcbtchenko. Notons que l'erreur est en partie excusable : le Nominatif du nom propre Juš̌enkoo (transcrit en français Youchtchenkoo) n'est pas marqué, car les noms de famille ukrainiens en $-\mathrm{o}$ sont indéclinables. Cela montre, une fois de plus, l'importance de la flexion nominale.

\subsection{Marquage du thème frontal par l'intonation et possibilités combinatoires}

En cas d'intonation dite neutre, l'élément thématique est placé en tête, alors que le dernier mot du rhème est normalement frappé par l'accent de phrase (dont la définition n'est pas sans poser des problèmes, cf. Nikolaeva, 2000, p. 70-73). Au niveau prosodique, le locuteur peut faire une légère pause (non obligatoire) entre le thème ou le rhème. Normalement, on marque par l'intonation la «rupture » entre le thème et le rhème : sur la syllabe accentuée du thème, on a un ton ascendant (de type IK-3 selon les modèles intonatifs élaborés par E. Bryzgunova, noté ici $\nearrow$ ), suivi d'une chute sur la syllabe post-tonique.

Ensuite, on a une baisse du ton (de type IK-1, noté ici〉) sur la syllabe accentuée du mot du rhème qui porte l'accent de phrase (voir Bryzgunova, 1980 ; Janko, 2001, p. 26-27, et bibliographie).

Soit une phrase à trois termes (sujet, prédicat verbal, COD). Avec une intonation dite neutre, on peut envisager 5 possibilités "canoniques », grâce à la permutation des termes et au découpage thémo-rhématique noté par //: 
(22) Ivan \// budit sýna \

Th $\mathrm{Rh}$

'Ivan réveille (son) fils'

(comme réponse à la question «Que fait Ivan ?»)

(23) Ivan \budit // sýna \

Th Rh

'Ivan réveille (son) fils'

(comme réponse à la question «Qui Ivan réveille-t-il ? »)

(24) Budit synaノ// Iván \

Th $\quad \mathrm{Rh}$

'Celui qui réveille (son) fils c'est Ivan'

(comme réponse à la question «Qui réveille le fils ? »)

(25) Syna \ budit // Iván \

Th $\quad \mathrm{Rh}$

'Celui qui réveille (son) fils c'est Ivan'

(comme réponse à la question «Qui réveille le fils ? ") 'Ivan, (son) fils, il le réveille'

(26) Ivan syna $\nearrow / /$ buidit $\searrow$

Th $\quad \mathrm{Rh}$

(comme réponse à la question «Que fait Ivan par rapport à son fils ? »)

Précisons que dans (23) c'est le sujet Ivan qui porte l'accent et se caractérise par le ton ascendant IK-3, alors que le prédicat budit constitue l'élément atone du thème. On a une situation analogue dans (25) où le ton ascendant caractérise le COD syna. Pour que budit soit accentué et marqué du ton IK-3, il faudrait un contraste :

(23') Ivan budit // s'́na \, a ukladyvaet - vnuka

'(D’habitude,) Ivan réveille (son) fils et (par ailleurs) il couche son petit-fils'

(25’) Syna budit \ // Iván \, a ukladyvaet - Maša

'(D’habitude,) celui qui réveille (son) fils c'est Ivan, et / alors que c'est Macha qui le couche'

En revanche, il serait difficile d'avoir (hors contextes de contraste) :

(27) ??Budit $/ /$ syna Iván \

Th $\quad \mathrm{Rh}$

?? 'Quant à l'action de réveiller, c'est Ivan [qui le fait] et c'est (son) fils [qui est concerné]' 
(28) ?Pynaノ// budit Iván】

Th $\quad \mathrm{Rh}$

?? 'Quant au fils, il y a qu'Ivan le réveille'

En effet, il est improbable d'imaginer des contextes qui permettent de poser des questions du genre «Quels sont les actants de l'action de réveiller dans la situation décrite ? », « De quelle action et faite par qui le fils fait-il l'objet? ». Mais (28) devient possible s'il y a contraste.

Notons qu'en dehors de la syntaxe standard (celle du russe écrit ou de l'écrit oralisé), la langue orale non standard présente des structures plus complexes: structures disloquées à reprise anaphoriques analogues à celles du français courant :

Syn Ivána // niktó \ ego ne budit

Th $\quad \mathrm{Rh}$

'Le fils d'Ivan, personne ne le réveille' (syn étant au Nominatif)

Mais on peut avoir un modèle intonatif un peu différent, avec IK-2 sur le prédicat ne budit.

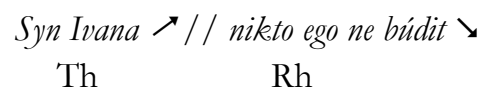

\subsection{Autres schémas intonatifs}

Parfois, le thème frontal peut être marqué dans le russe oral spontané par un ton ascendant de type IK-6 symbolisé par $\Gamma$. A la différence de IK-3 qui se caractérise par une chute du ton sur les syllabes post-accentuées, on a ici le maintien du ton soutenu sur les syllabes post-accentuées (Janko, 2001, p. 27). Cf. :

(30) Nedavno $/ / /$ \# prixodil Ivan $\downarrow$

Th $\quad \mathrm{Rh}$

'Il n'y pas longtemps / Récemment, Ivan est venu'

(31) Nedavno $\Gamma / /$ prixodil Ivan

Th $\quad \mathrm{Rh}$

'Il n'y pas longtemps / Récemment, Ivan est venu'

Si dans (30) on peut faire une pause entre le thème et le rhème, le modèle intonatif IK-6 rend la pause (symbolisée par \#) impossible dans (31). En réalité, c'est la chute brusque du ton après la syllabe accentuée dans IK-3 qui peut créer l'illusion d'une pause, alors dans IK-6 il n'y a pas de chute.

Cependant, cette marque prosodique caractérise également le rhème et elle ne fonctionne pas indépendamment du sémantisme de la séquence qu'elle modifie. Cf. (exemple de monorhème) :

Davno $\longmapsto$ ne prixodil Ivan $\downarrow$

$\mathrm{Rh}$

'Cela fait longtemps qu'Ivan n'est pas venu’ 
Davno ne peut fonctionner qu'au sein d'un rhème, alors que nedavno peut faire partie d'un thème ou d'un rhème.

Le ton IK-6 peut caractériser le dernier des thèmes au cas où il y en a plusieurs (Janko, 2001, p. 129) :

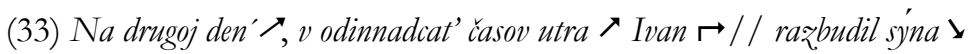

$\begin{array}{llll}\text { Th1 Th2 Th3 } & \text { Th }\end{array}$

'Le lendemain, à onze heures du matin, Ivan // a réveillé son fils'

Il existe un marquage particulier en cas de thème "de contraste » (kontrastnaja tema), lorsqu'il y a une opposition explicite ou implicite : le modèle intonatif est de type IK-3-K, il s'agit d'un ton plus soutenu que celui de IK-3, ce ton est noté par une double flècheノノ (Janko, 2001, p. 32) :

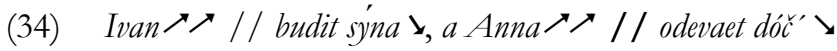

Th Rh Th Rh

'Ivan réveille (son) fils, alors qu'Anna habille (sa) fille'

Notons que (34) est possible p. ex. dans une situation où on pose une question du genre «Que font Ivan et Anna ce matin?».

Le rhème lui aussi peut être marqué par un ton « de contraste »: IK-1-K 〉〉

Iz amerikanskix vin イハ // on predpočitaet kalifornijskie ১১

$\mathrm{Th}$

$\mathrm{Rh}$

'Quant aux vins américains, il préfère ceux de Californie',

mot à mot « Des vins américains / / il préfère les californiens ».

Le contraste s'établit ici par rapport aux autres vins connus présents sur le marché (vins français, vins allemands, vins espagnols, vins italiens, vins australiens, etc.).

\section{Cas de thème non frontal}

\subsection{Thème final en syntaxe dite expressive: intonation emphatique sur le rhème}

Dans les modèles syntaxiques dits expressifs, le thème est souvent en deuxième position, alors que le rhème est en tête. Le rhème est marqué d'une intonation emphatique IK-1-E symbolisée par $\Lambda$, qui est en partie analogue à la construction intonative IK-1 du point de vue du mouvement mélodique (ton descendant sur la syllabe accentuée) mais se caractérise par une amplitude plus importante mais surtout par un contour « recourbé» (iskrivlënnyj), puisque la phase descendante est précédée d'une hausse (Janko, 2001, p. 119):

(36) Iván $\Lambda$ // budit syna \

$\mathrm{Rh} \quad \mathrm{Th}$

'C'est bien Ivan qui réveille (son) fils' 


\subsection{Thème atonique dit « récessif » ou « de Wackernagel »}

Un cas très fréquent est à signaler avec le rhème frontal marqué du ton IK-1 suivi d'un thème atonique (Janko, 2001, p. 207) :

$$
\text { [Priš̈l bol'noj k vraču.] Osmotrél \// vrač bol'nogo }
$$

$\mathrm{Rh}$

ThA

'[Un malade est venu chez un médecin.] Le médecin a examiné le malade', mot à mot «Examina (Passé perf. $3^{e}$ pers. sing. masc.) // médecin (Nominatif sing. masc.) malade (Accusatif animé sing. masc.) »

En effet, ce thème (noté ThA) n'est marqué dans ce cas d'aucun ton, c'est-àdire il est prononcé sur un ton égal, d'une façon rapide et «incolore ». Il s'agit d'un thème « récessif » (recessivnaja tema), dit « de Wackernagel » (Janko, 2001, p. 79-80, 205), par référence à la célèbre loi de J. Wackernagel (1892) dans son interprétation élargie, loi selon laquelle les enclitiques occupaient dans les langues indo-européennes anciennes la deuxième position dans une phrase, se plaçant après le premier mot à sens plein accentué.

On a un découpage thème-rhème particulier avec dislocation du rhème, sans possibilité de pause, dans une narration imitant le style oral (genre anecdote, conte populaire) :

\section{Prixodit $\sqcap / /$ Ivan // domoj $\downarrow$}

$\mathrm{Rh} \quad \mathrm{ThA} \quad \mathrm{Rh}$

'Et v'là qu'Ivan rentre chez lui', mot à mot «Vient Ivan à la maison »

Budit $\mapsto / /$ Ivan // syna

$\mathrm{Rh} \quad \mathrm{ThA} \quad \mathrm{Rh}$

'Et v'là qu'Ivan réveille son fils', mot à mot « Réveille Ivan fils »

La partie frontale du rhème est marquée du ton IK-6, alors que le thème est atonique.

\section{Thématisation et mots du discours}

Le russe dispose de nombreux mots du discours, appelés traditionnellement «particules », qui jouent un rôle considérable dans la communication orale. A première vue, les mots discursifs qui marquent le rhème (imenno, kak ra‡, èto, ved', dǎ̌̀, i) sont plus nombreux que les marqueurs du thème (-to, ‘̌e), ces derniers d'ailleurs n'étant pas des marqueurs thématiques spécifiques, puisqu'ils apparaissent aussi dans le rhème sous certaines conditions syntaxiques. Mais de façon générale, plusieurs particules fréquentes comme le «rhématique » daže 'même' ou le «thématique» ž้e (traduit parfois inexactement par donc dans les dictionnaires) peuvent se rapporter tantôt au rhème, tantôt au thème. Cf. le double fonctionnement de žze: 


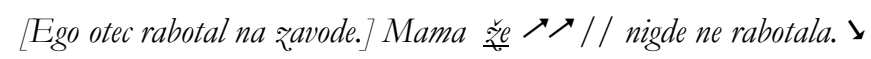

Th

$\mathrm{Rh}$

'Son père travaillait à l'usine. Quant à (sa) mère, elle ne travaillait pas'

$$
\begin{aligned}
& \text { [Ty čto sidiš́ ?] Máma } \Lambda_{\text {že nas zovët! }} \\
& \text { Rh (monorhème) }
\end{aligned}
$$

'[Qu'est-ce que tu as à rester assis ? ] Il y a maman qui nous appelle !'

La particule -to (remontant au pronom-adjectif démonstratif to) marque souvent le thème dans la langue familière (Bonnot, 1991) avec un effet de sens particulier (par exemple, le locuteur veut exprimer sa surprise en mettant en rapport l'actant correspondant au thème avec l'événement constaté). Le thème peut être marqué du ton «recourbé » emphatique IK-3-E (Janko, 2001, p.119), alors que le rhème peut porter un ton « recourbé » emphatique IK-1-E, cf.

$$
\text { Iván-to } \mathrm{V} / / \text { sýna } \wedge \text { budit! }
$$

Th $\mathrm{Rh}$

'Tiens, v'là Ivan qui réveille (son) fils! [Le temps va changer !]', mot à mot «Ivan-TO // fils (Accusatif animé masculin sing.) réveille ! »

L'effet de sens induit par la particule -to est le suivant: ce qu'on sait sur Ivan est questionné du point de vue de sa compatibilité avec l'événement « réveiller son fils » : par exemple, le locuteur veut exprimer sa surprise par rapport à l'événement, compte tenu de ce qu'il sait sur les habitudes d'Ivan (normalement, Ivan s'abstient de réveiller son fils, puisque c'est sa femme qui le fait ; de toute façon, Ivan ne s'occupe pas souvent de ses enfants, etc.)

L'accent de phrase sera sur budit si le locuteur dispose d'un savoir préalable qui est susceptible de remettre en cause l'action de « réveiller » : par exemple, si l'on sait qu'Ivan dit souvent à tout le monde qu'on ne doit pas réveiller les enfants, qu'il faut les laisser dormir pour ne pas perturber leur croissance.

\section{Conclusion}

A première vue, le marquage du thème s'effectue souvent par défaut, puisque c'est plutôt le rhème qui apparaît comme le segment linguistiquement marqué, du moins en cas d'intonation dite neutre dans le russe standard : position finale dans la phrase, à gauche du thème, accent de phrase. Au contraire, le thème peut être atonique, intonativement «incolore ». Enfin, le thème semble correspondre à la partie la moins importante car la moins informative de l'énoncé : en effet, dans certains énoncés (cas de monorhèmes), le thème n'est même pas exprimé, alors que le rhème est nécessairement exprimé.

Il semblerait même «naturel» que le rhème, qui est le segment considéré souvent comme le plus important du point de vue de la communication, soit le terme marqué de l'opposition. On va en effet du «connu» au « nouveau » : normalement, le « nouveau » est logiquement en position finale, en position «forte» du point de vue 
communicationnel. En termes plus théoriques, selon l'approche dite de "perspective fonctionnelle de la phrase » due au second Cercle de Prague (Daneš, 1974), les places de début (thème), du milieu (transition) et de fin de phrase (rhème) possèdent un degré différent de dynamisme communicatif : du plus bas degré pour le thème au plus haut degré pour le rhème (Lane, 2002, p. 573).

Mais la réalité discursive est plus complexe : le thème, lorsqu'il existe, apparait au contraire comme un constituant essentiel dans la construction de l'énoncé, car il fournit un cadre à la relation prédicative (Bonnot, 1991, p. 30). Le marquage du thème en russe est subtil et varié, même si les marques en question ne fonctionnent pas indépendamment de celles du rhème (tout d'abord au niveau prosodique).

Il reste dans ce domaine des points insuffisamment étudiés, surtout si on tient compte de la langue orale spontanée non standard - même si cette dernière est moins présente dans l'enseignement, il est vrai, que la langue écrite standard, puisque même à l'oral, on enseigne l'écrit oralisé en ayant tendance à exclure toute structure «qui fait trop parlé ». 


\section{BIBLIOGRAPHIE}

Adamczewski H., 1991, Le français déchiffré, A. Colin, Paris.

BENOIST J.-P., 1979, Les fonctions de l'ordre des mots en russe moderne, Institut d'Etudes slaves, Paris.

Bonnot Ch., 1991, La particule de thématisation - to en russe moderne. Thèse de doctorat d'Etat, Université Paris IV, Paris.

BonnOT Ch., 1999, «Pour une définition formelle et fonctionnelle de la notion de thème (sur l'exemple du russe moderne) », in : Cl. Guimier (éd.), 1999 (voir infra), p. 15-32.

BONNOT Ch., 2004, «Relation préconstruite et focalisation: pour une analyse unitaire des énoncés à accent non final en russe moderne », Slovo, 30/31, p. 211-271.

Boulanger A., 2000, Pratique de la traduction russe: Eléments de syntaxe comparée, Ophrys, Paris, Gap.

Bryzgunova E. A., 1980, «Intonacija », - in : Švedova N. Ju. (dir.), Russkaja grammatika, T. 1, Nauka, Moskva, p. 96-122.

Chafe W. L., 1976, "Giveness, contrastiveness, definiteness, subjects, topics and point of view », - in: Ch. Li (ed.), Subject and Topic, Academic Press, New York, p. 25-55.

Comtet R., 2002, Grammaire du russe contemporain, 2e éd., Presses Universitaires du Mirail, Toulouse.

DANES F. (éd.), 1974, Papers on functional sentence perspective, Mouton, The Hague.

Fougeron I., 1989, Prosodie et organisation du message: Analyse de la phrase assertive en russe contemporain. Klincksieck, Paris.

Fougeron I., 2004, "Focalisation du thème en russe contemporain », Bulletin de la Société de Linguistique de Paris, XCIX, fasc. 1.

GAK V. G., 1989, Sravnitel'naja tipologija francu々skogo i russkogo jarylkov, Prosveščenie, Moskva.

Guimier Cl. (éd.), 1999, La thématisation dans les langues. Actes du colloque de Caen 9-11.10.97, P. Lang, Bern.

JANKO T. E., 2001, Kommunikativnye strategii russkoj reči, Jazyki russkoj kul'tury, Moskva.

Kiseleva K., PailLard D. (eds), 1998, Diskursivnye slova russkogo jasyka, Metatekst, Moskva.

Kovtunova I. I., 1976, Sovremennyj russkij jasylk. Porjadok slov i aktual'noe členenie predloženija, Prosveščenie, Moskva.

Kovtunova I. I., 1980, «Porjadok slov», in : Švedova N. Ju. (dir.), Russkaja grammatika, T. 2, Nauka, Moskva, p. 190-214; 257-269.

LANE Ph., 2002, "Thème / rhème », in : P. Charaudeau, D. Maingueneau (dir.), Dictionnaire d'analyse du discours, Seuil, Paris, p. 572-573.

MarChello-Nizia Ch., 1999, Le français en diachronie : douže siècle d'évolution, Ophrys, Paris. 
Thématisation dans les langues flexionnelles, le cas du russe

Nikolaeva T. M., 2000, Ot zvuka k tekstu, Jazyki russkoj kul'tury, Moskva.

SAKHNO S., «Les formes de l'adjectif attribut en russe: prédication "effectuée" versus prédication "mentionnée" »-La Revue des études slaves (Paris), t. 73, fasc. 1, 2001, pp. 77-96.

SITRI F., 2003, L'objet du débat. La construction des objets de discours dans des situations argumentatives orales, Presses Sorbonne Nouvelle.

WACKERNAGEL J., 1892, «Über ein Gesetz der indogermanischen Wortstellung », - Indogermanische Forschungen, 1, p. 333-436.

WEIL H., [1844] 1991, De l’ordre des mots dans les langues anciennes comparées aux langues modernes, Didier, Paris.

Zolotova G. A., 1982, Kommunikativnye aspekty russkogo sintaksisa, Nauka, Moskva. 
